Dokufiktionalität in Literatur und Medien 



\section{Dokufiktionalität in Literatur und Medien}

Erzählen an den Schnittstellen von Fakt und Fiktion

Herausgegeben von

Agnes Bidmon und Christine Lubkoll

\section{DE GRUYTER}


Die freie Verfügbarkeit der E-Book-Ausgabe dieser Publikation wurde durch 39 wissenschaftliche Bibliotheken ermöglicht, die die Open-Access-Transformation in der Deutschen Literaturwissenschaft fördern.

ISBN 978-3-11-069298-3

e-ISBN (PDF) 978-3-11-069299-0

e-ISBN (EPUB) 978-3-11-069308-9

DOI https://doi.org/10.1515/9783110692990

\section{(cc) BY}

Dieses Werk ist lizenziert unter der Creative Commons Attribution 4.0 International (CC BY 4.0) Lizenz. Weitere Informationen finden Sie unter http://creativecommons.org/licenses/by/4.0/.

Library of Congress Control Number: 2021947406

\section{Bibliografische Information der Deutschen Nationalbibliothek}

Die Deutsche Nationalbibliothek verzeichnet diese Publikation in der Deutschen Nationalbibliografie; detaillierte bibliografische Daten sind im Internet über http://dnb.dnb.de abrufbar.

(c) 2022 bei den Autorinnen und Autoren; Konzeption und Herausgabe (c) 2022 Agnes Bidmon und Christine Lubkoll, publiziert von Walter de Gruyter GmbH, Berlin/Boston Dieses Buch ist als Open-Access-Publikation verfügbar über www.degruyter.com.

Umschlaggestaltung: Trokantor / E+ / gettyimages

Satz: le-tex publishing services $\mathrm{GmbH}$, Leipzig

Druck und Bindung: CPI books $\mathrm{GmbH}$, Leck

www.degruyter.com 


\section{Open-Access-Transformation in der Literaturwissenschaft}

Open Access für exzellente Publikationen aus der Deutschen Literaturwissenschaft: Dank der Unterstützung von 39 wissenschaftlichen Bibliotheken können 2021 insgesamt neun literaturwissenschaftliche Neuerscheinungen transformiert und unmittelbar im Open Access veröffentlicht werden, ohne dass für Autorinnen und Autoren Publikationskosten entstehen.

Folgende Einrichtungen haben durch ihren Beitrag die Open-Access-Veröffentlichung dieses Titels ermöglicht:

Universitätsbibliothek Augsburg

Universitätsbibliothek Bayreuth

University of California, Berkeley Library

Staatsbibliothek zu Berlin - Preußischer Kulturbesitz

Universitätsbibliothek der Freien Universität Berlin

Universitätsbibliothek der Humboldt-Universität zu Berlin

Universitätsbibliothek Bielefeld

Universitäts- und Landesbibliothek Bonn

Universitätsbibliothek Braunschweig

Staats- und Universitätsbibliothek Bremen

Universitätsbibliothek der Technischen Universität Chemnitz

Universitäts- und Landesbibliothek Darmstadt

Sächsische Landesbibliothek - Staats- und Universitätsbibliothek Dresden

Universitätsbibliothek Duisburg-Essen

Universitäts- und Landesbibliothek Düsseldorf

Universitätsbibliothek Johann Christian Senckenberg, Frankfurt a. M.

Bibliothek der Pädagogischen Hochschule Freiburg

Niedersächsische Staats- und Universitätsbibliothek Göttingen

Universitätsbibliothek Greifswald

Universitätsbibliothek der FernUniversität in Hagen

Universitäts- und Landesbibliothek Sachsen-Anhalt, Halle (Saale)

Staats- und Universitätsbibliothek Hamburg Carl von Ossietzky

Gottfried Wilhelm Leibniz Bibliothek - Niedersächsische Landesbibliothek, Hannover

Universitäts- und Landesbibliothek Tirol, Innsbruck

Universitätsbibliothek Kassel - Landesbibliothek und Murhardsche Bibliothek der Stadt Kassel Universitätsbibliothek der Universität Koblenz-Landau

Zentral- und Hochschulbibliothek Luzern

Universitätsbibliothek Marburg

Universitätsbibliothek der Ludwig-Maximilians-Universität München

Universitäts- und Landesbibliothek Münster

Bibliotheks- und Informationssystem der Carl von Ossietzky Universität Oldenburg

Landesbibliothek Oldenburg

Universitätsbibliothek Osnabrück

Universitätsbibliothek Trier

Universitätsbibliothek Vechta

Herzogin Anna Amalia Bibliothek, Weimar

Herzog August Bibliothek Wolfenbüttel

Universitätsbibliothek Wuppertal

Zentralbibliothek Zürich 
